\title{
Plasma from volunteers breathing helium reduces hypoxia induced cell damage in human endothelial cells
}

\author{
Kirsten F Smit ${ }^{1}$, Gezina TML Oei ${ }^{1}$, Markus W Hollmann ${ }^{1}$, Benedikt Preckel ${ }^{1}$, Hemal Patel ${ }^{2}$, Nina C Weber ${ }^{1}$ \\ ${ }^{1}$ Department of Anaesthesiology, Laboratory of Experimental Intensive Care and Anaesthesiology (L.E.I.C.A.) \\ Academic Medical Center (AMC), University of Amsterdam, The Netherlands
}

${ }^{2}$ Veterans Affairs San Diego Healthcare System

\section{Hypothesis \& Background}

Remote ischemic preconditioning, induced by short repetitive ischemic stimuli in one organ, can protect another organ from prolonged ischemia/reperfusion. Plasma taken from volunteers subjected to $4 \times 5$ minutes of forearm ischemia protects endothelial cells in vitro against hypoxia-induced damage. In vivo, preconditioning with helium protected healthy volunteers against post-ischemic endothelial dysfunction. The mechanism behind helium preconditioning is not yet clear. Here we investigate whether plasma from helium treated volunteers in vivo can protect human umbilical vein endothelial cells (HUVEC) remotely against hypoxia.

\section{Methods}

After ethical approval, 20 healthy male volunteers inhaled heliox ( $79 \%$ helium, $21 \%$ oxygen) or air for 30 minutes, in a crossover design with two weeks between experiments. Plasma was collected at baseline (T0), directly after inhalation (T1), 6h (T3) and 24h (T4) after start of the experiment. HUVEC were incubated with either $5 \%$ or $10 \%$ of the plasma for 1-2 hours and were subsequently subjected to enzymatically induced hypoxia for 24 hours. Cell damage was measured by LDH content of supernatant and cellular fractions. Caveolin-1 (Cav-1), hypoxic inducible factor (HIF) $1 \alpha$, extracellular signal-regulated kinase (ERK) $1 / 2$, signal transducer and activator of transcription (STAT) 3 and endothelial nitric oxide synthase (eNOS) were determined by Western blot analysis and enzyme-linked immuno sorbent assay (ELISA). Additional experiments were performed using siRNA cav-1 transfected HUVEC $(n=10)$.
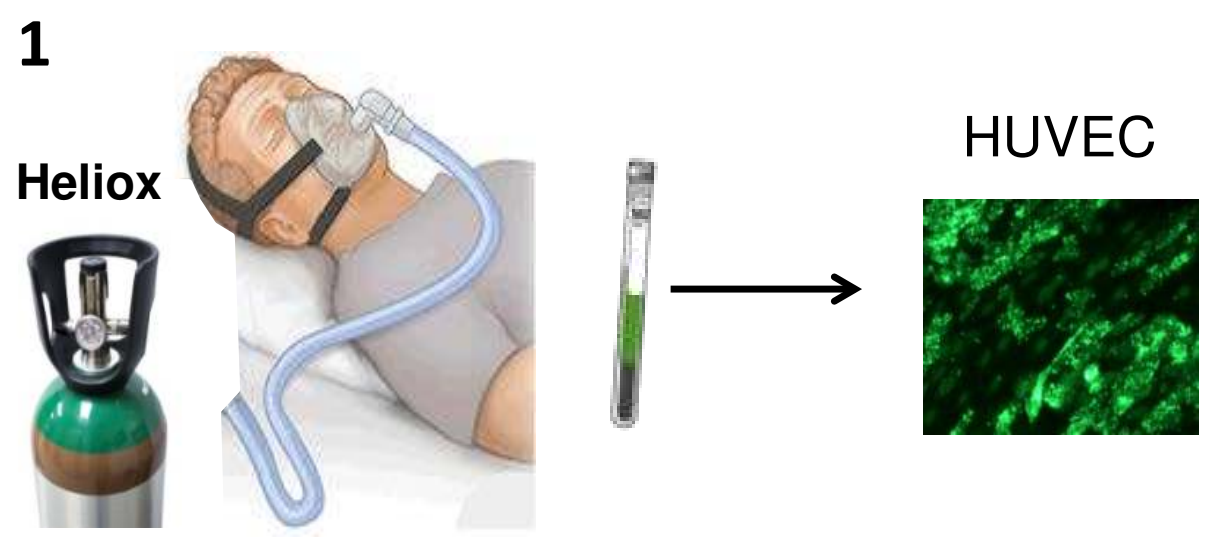

Protection against hypoxia?

\section{Results 1: LDH}

2 Relative LDH Activity
Figure 2

Prolonged pre-hypoxic exposure to $10 \%$ plasma obtained 6 hours after helium inhalation significantly decreased hypoxia induced cell damage in HUVEC compared to baseline.

Mean 95\% Cl 0,86 (0,77;0.95). $\mathrm{TO}=1$.
Results 2: Caveolin
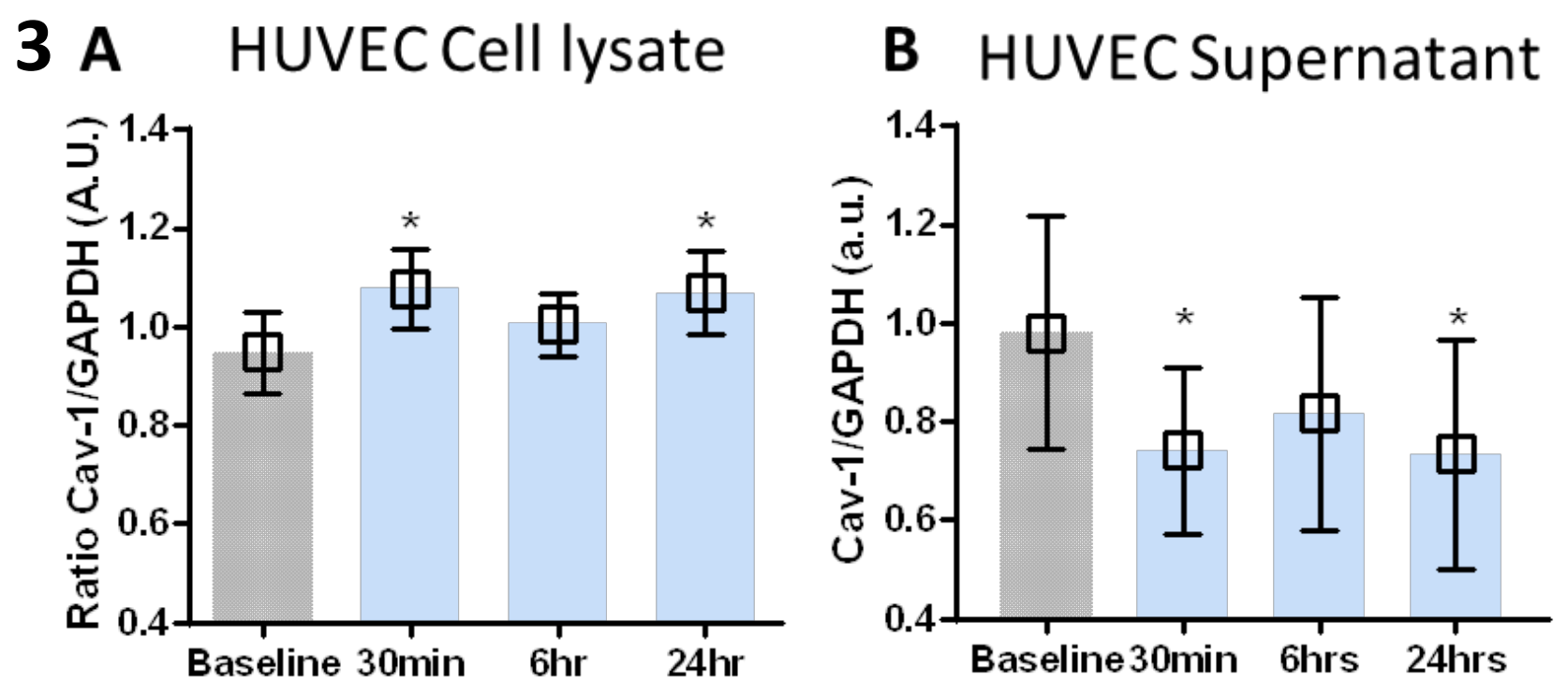

Columns represent means $( \pm 95 \% \mathrm{Cl}) .^{*}=p<0,05$ to baseline.

Figure 3. Plasma obtained directly after (T1) and $24 \mathrm{~h}$ after (T3) helium inhalation caused a significant increase in cytosolic Cav-1 levels $\mathrm{T} 0=0.95[0.86,1.03], \mathrm{T} 1=1.1[0.99,1.16]$ and $\mathrm{T} 3=1.0[0.98-$ 1.15] respectively, $p<0.05$. In conjunction with increased cellular levels of Cav-1, we found decreased levels in the supernatant corresponding time points $\mathrm{T} 1$ and $\mathrm{T} 3$ compared to baseline $\mathrm{T} 0=1.0$ $[0.74,1.20], \mathrm{T} 1=0.8[0.59,1.02]$ and $\mathrm{T} 3=0.7[0.50,0.96], \mathrm{p}<0.05)$.

Results 3: cav-1 transfection

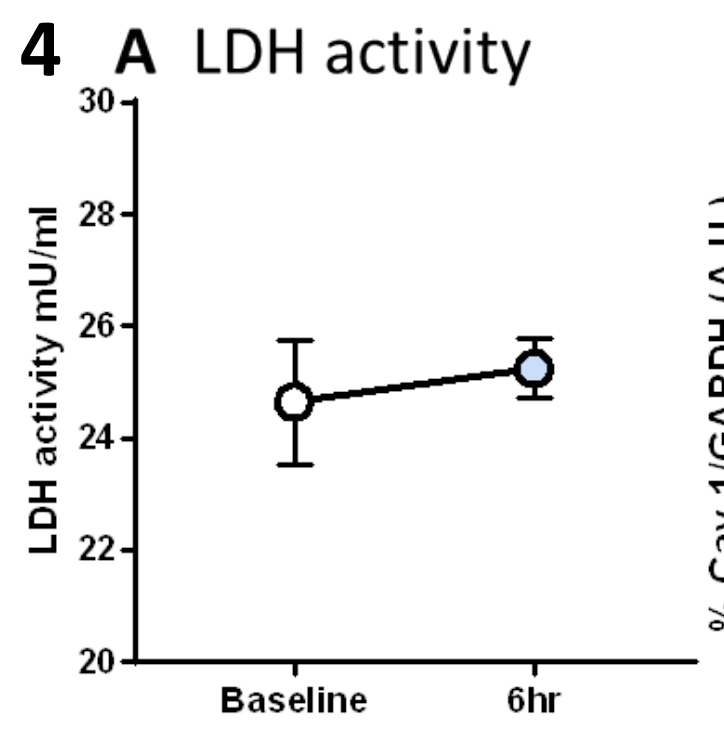

B Caveolin 1 levels

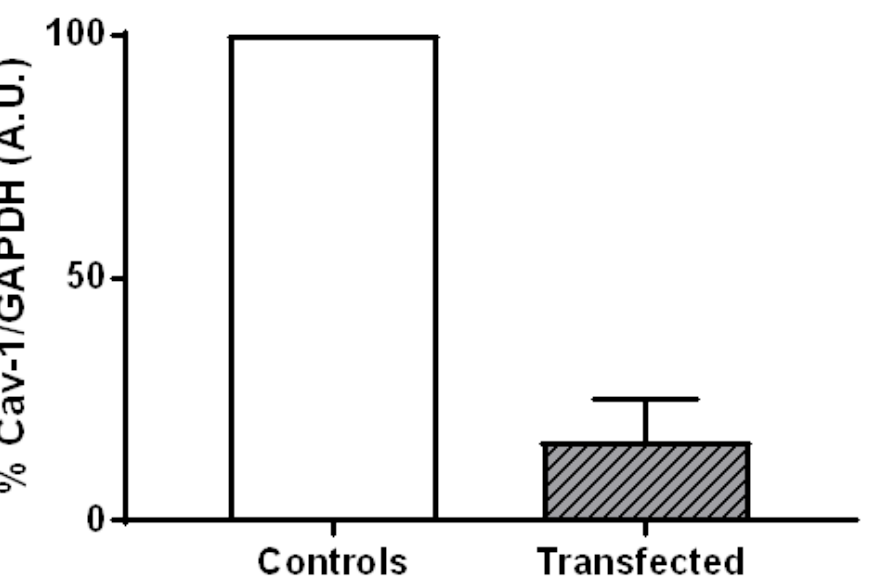

Figure 4. Transfection with Cav-1 siRNA successfully lowers Cav-1 levels in HUVEC to $16 \%$ compared to HUVEC transfected with negative control siRNA. In Cav-1 siRNA transfected HUVEC, the protective effect of T2 plasma was abolished, showing no difference between LDH in cells treated with baseline (TO) plasma $(25,5[24,7$, $25,8]$ and $24,6[23,5,25,7])$ respectively. (means [95\% CI]). This indicates cav- 1 possibly plays a role in helium remote protection against hypoxia.

\section{Conclusion}

Plasma of healthy volunteers breathing helium protects HUVEC against hypoxic cell damage. Cav-1 knockdown in siRNA transfected HUVEC abolished helium remote preconditioning, indicating caveolin-1 mediates helium remote preconditioning. 